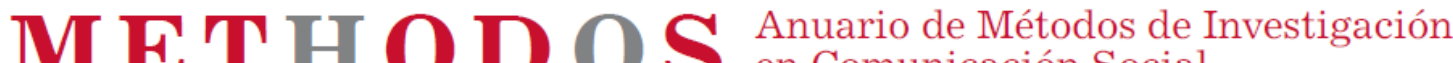 en Comunicación Social
}

Mayo 2020

\section{Los métodos etnográficos y los fan studies, un cambio de paradigma}

\author{
Ona Anglada Pujol \\ Universitat Pompeu Fabra \\ ona.anglada@upf.edu
}

DOI: 10.31009/methodos.2020.i01.09

Anglada-Pujol, O. (2020). Los métodos etnográficos y los fan studies, un cambio de paradigma. En: Lopezosa, C.; Díaz-Noci, J.; Codina, L. (ed.). Anuario de Métodos de Investigación en Comunicación Social, n.1 (p.98-105). Barcelona: DigiDoc-Universitat

Pompeu Fabra

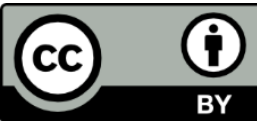





\title{
Los métodos etnográficos y los fan studies, un cambio de paradigma
}

\author{
Ona Anglada Pujol \\ Universitat Pompeu Fabra \\ ona.anglada@upf.edu
}

\section{RESUMEN}

En este artículo se presentan varios de los debates y cuestiones alrededor de metodología etnográfica aplicada a los fan studies. Esta disciplina, aparecida en los años noventa, usa principalmente métodos etnográficos y análisis de contenido. Hemos revisado los cambios en la concepción del fan y como esto se ha reflejado en la metodología, centrándonos en los fans de cultura mediática popular y no en los fans de deportes. Se ha realizado una revisión de los artículos sobre metodologías aplicadas en los fan studies y una síntesis de todas aquellas cuestiones surgidas a raíz de varias problemáticas, como la aparición de las comunidades de fans online. También se revisan conceptos como "aca-fan" y las implicaciones que este tiene en las aproximaciones etnográficas.

\section{PALABRAS CLAVE}

Fan studies, etnografía, aca-fan, metodología, etnografía digital.

\section{Ethnographic methods and fan studies, a paradigm shift}

\section{Els mètodes etnogràfics i els fan studies, un canvi de paradigma}

\section{ABSTRACT}

In this paper we present various debates and issues around the ethnographic methodology used in fan studies. This field, which appeared in the nineties, basically uses ethnographic methods and content analysis. We've revised the changes in the understanding of fan and how these changes have been reflected in the methodology, putting our focus on the pop fandom and not in the sport fandom. We've made a revision of the papers about methodologies used in fan studies and a synthesis of all the questions that arise from several issues, like the emergence of online fan communities. We also revise concepts like aca-fan and the consequences that has in the ethnographic approaches.

\section{KEYWORDS}

Fan studies, ethnography, aca-fan, methodology, digital ethnography

\section{RESUM}

En aquest article es presenten varis dels debats i qüestions al voltant de la metodologia etnogràfica aplicada als fan studies. Aquesta disciplina, apareguda als anys noranta, utilitza principalment mètodes etnogràfics $i$ anàlisis de contingut. Hem revisat els canvis en la concepció del fan i com aquests s'han reflectit en la metodologia, centrant-nos en els fans de cultura mediàtica popular i no en els fans d'esports. S'ha realitzat una revisió dels articles sobre metodologies aplicades als fan studies i una síntesis de totes aquestes qüestions sorgides arran de vàries problemàtiques, com l'aparició de comunitats de fans online. També es revisen conceptes com "aca-fan" i les implicacions que té en les aproximacions etnogràfiques.

\section{PARAULES CLAU}

Fan studies, etnografia, aca-fan, metodologia, etnografia digital. 


\section{Introducción}

El presente artículo quiere hacer una fotografía de las principales cuestiones, debates y retos alrededor de las metodologías etnográficas que se aplican en los fan studies. Aunque no es la única metodología que se utiliza - el análisis de contenido también se usa habitualmente (Evans and Stasi 2014, 17) - nos centraremos en los métodos etnográficos, ya que son aquellos con más tradición dentro de los fan studies pero que a la vez más han cambiado.

Debido a la aparición de las comunidades de fans online y un cambio en la concepción de la figura del investigador han generado que se haya replanteado y debatido las formas de aproximarse a los fans desde la perspectiva etnográfica.

En esta revisión nos hemos centrado especialmente en todos esos artículos o investigaciones focalizadas en tratar temas de metodología, especialmente en esos que hablasen de métodos etnográficos. De todas formas, también se han consultado libros y artículos de referencia de la disciplina (como los libros de Henry Jenkins o Camille Bacon-Smith), así como investigaciones que hayan utilizado esta metodología, para ver las tendencias y aplicaciones.

Los fan studies, según la definición de Jenkins (2006a), son "a field of scholarly research focused on media fans and fan cultures. Fans might be broadly defined as individuals who maintain a passionate connection to popular media, assert their identity through their engagement with and mastery over its contents, and experience social affiliation around shared tastes and preferences".

En esta disciplina se entiende a los fans como una audiencia activa y que se apropia de la cultura mediática, de forma que "the field of fan studies offers the ultimate rebuttal of the traditional media effectsmodel, by stressing the relative independence, agency and power of media consumers vis-à-vis media producers" (Reijnders et al. 2016, 1-2).

A continuación, veremos las diferentes maneras de entender los fans y sus actividades, así como la relación entre investigador y fandom. Todas estas cuestiones nos llevan a ver por qué se usan los métodos etnográficos y de qué forma, ya que la elección de cierta metodología o cómo se aplica esta va muy ligada a la propia concepción de los fans.

\section{Los fan studies: definiendo la disciplina}

Antes de analizar al detalle los métodos utilizados en los fan studies, debemos fijarnos en algunas cuestiones básicas. En primer lugar, tenemos que diferenciar los estudios que se centran en los fans de cultura pop y los que se centran en los fans de deportes. Aunque podría parecer que funcionan de manera similar, veremos que hay muchas diferencias entre los dos campos. Otra cuestión importante es la interdisciplinariedad de los fan studies, que tiene una repercusión a nivel metodológico, pero también de la misma concepción de qué es un fan y cómo debemos aproximarnos al fenómeno. También será relevante fijarnos en cuándo y de dónde nacieron los fan studies, para entender mejor las causas y orígenes de esta interdisciplinariedad.

En este artículo nos centraremos solamente en los fans de la cultura mediática popular, sin entrar en cuestiones relacionadas con los fans de los deportes. Esta distinción la haremos básicamente porque tanto los ámbitos desde los que se estudian ambos fenómenos, como también en los métodos que se usan, hay una gran disyuntiva, que ha hecho que "these two literatures have developed on separate trajectories and occupy quite different spaces in the scholarly field" (Schimmel, Harrington, and Bielby 2007, 583).

Aunque las dos disciplinas usan habitualmente una aproximación etnográfica, los sport fan studies tienden a utilizar métodos cuantitativos, especialmente cuando se estudia desde una perspectiva psicológica. Así pues, toda la literatura revisada para este artículo se centra en la metodología de los pop fan studies, sin poner el foco en otros métodos que podrían emplearse para analizar otro tipo de fans.

No obstante, autores como Schimmel, Harrington y Bielby, apuestan por un acercamiento entre los dos campos que genere intercambios y los amplíe: "Sport fan scholars and pop culture fan scholars gain significantly through greater cross-fertilization of ideas and knowledge - from talking with, rather than past, each other" $(2007,593)$.

\section{Historia de los fan studies}

Lo más habitual es situar el inicio de los fan studies en la publicación del libro Textual Poachers: Television Fans \& Participatory Culture de Henry Jenkins el año 1992 (Reijnders et al. 2016) aunque ese mismo año también aparecieron dos libros muy relevantes para la disciplina: Enterprising Women de Camille Bacon-Smith y The Adoring Audience de Lisa Lewis. Los tres libros, publicados el 1992, surgieron en un momento en que los fans se consideraban marginales y también estaban "ridiculed in the media, shrouded with social stigma [...] and often depicted as brainless and inarticulate" (Jenkins 2006b, 1).

Estos tres autores provenían de tres campos muy diferentes: Henry Jenkins venía de los estudios 
culturales, Camille Bacon-Smith de los estudios de folklore y Lisa Lewis de estudios de música popular. Aún así, estos tres académicos empezaron a construir lo que ahora conocemos como los fan studies. Esta interdisciplinariedad se mantiene hasta el día de hoy (Harrington and Bielby 2005, 799), mezclando académicos de disciplinas como literatura inglesa, antropología, sociología, psicología, gender studies y estudios culturales, entre otros (Evans and Stasi 2014, $6)$.

Esto genera varias formas de entender el objeto de estudio y cómo acercarse a él, cosa que acaba condicionando las metodologías que se usan. Tal y como explica el mismo Jenkins, "fan studies began as the study of fan reception but has increasingly been recentered around forms of fan cultural production" (2006a). Estos primeros autores utilizaban básicamente métodos etnográficos para aproximarse a las comunidades de fans, con la intención de entender las formas que tenían éstas de relacionarse con los productos mediáticos y creando contenido propio que generaba otros significados (Ford 2014, 55).

Se considera que hay tres "olas" en los estudios de los fans, la primera ola tomó un enfoque más etnográfico y según Paul Booth, se centró en el fan como figura clave de las técnicas de resistencia subcultural $(2013,121)$. La segunda ola pasó de un "analysis of fan individual's consumption" a "larger issues of the fan community's social dynamics" $(2013,56)$. En esta etapa, el fandom se concebía como un elemento más dentro de las dinámicas jerárquicas y de poder de los medios y la sociedad, y los fans siendo otra pieza de este engranaje. Finalmente, la tercera ola se fija en las prácticas más cotidianas de los fans y cómo estos se han integrado en el sistema de medios, centrando no solo la atención en el fan sino en otros elementos del ecosistema mediático $(2013,57)$.
Es interesante prestar atención a estas tres olas y a las formas de entender a los fans de cada una de ellas, ya que acaban condicionando la metodología que se usa, moviéndose básicamente entre aproximaciones etnográficas o de análisis del discurso cualitativo (tabla 1).

Otro punto destacable es la poca cantidad de artículos y literatura dedicados específicamente a discutir metodologías dentro de los fan studies. Tal y como apuntan Adrienne Evans y Mafalda Stasi "despite a variety of published research in the area of fan studies, explicit reference to methodology or research methods was often missing" $(2014,5)$.

Las autoras creen que este hecho no es exclusivo y único de los fan studies, sino que podemos encontrarlo en disciplinas parecidas y casi 'hermanas', como los celebrity studies. Uno de los motivos que identifican como posible causa de este vacío de discusión y debate sobre metodología es la constante interacción de los fan studies con los estudios culturales. Los estudios culturales se caracterizan por ser una "anti-disciplina", poniendo el foco en la flexibilidad "with the aim of proceeding as a bricolage collective of methods, theories, ideas and concepts" (Evans and Stasi 2014, 8). Así pues, es lógico que los fan studies adopten las formas de los estudios culturales, que habitualmente rehúye encerrarse en una serie de métodos concretos (T. Bennett 1998, 530).

Podemos identificar dos metodologías principales en los fan studies: la etnográfica (principalmente observación participante y entrevistas en profundidad) y el análisis de contenido. En este artículo nos centraremos en los métodos etnográficos. Aunque hay muchos análisis que combinan las dos formas, el aproximamiento etnográfico es el que más ha variado y que más debates genera actualmente, con las etnografías digitales.

\begin{tabular}{|c|c|c|c|}
\hline & Primera ola & Segunda ola & Tercera ola \\
\hline \multirow[t]{3}{*}{ Concepción del fandom } & $\begin{array}{l}\text { Esfuerzo colectivo que genera } \\
\text { lecturas de resistencia. }\end{array}$ & $\begin{array}{l}\text { Un elemento más de jerarquías } \\
\text { culturales y políticas económicas. }\end{array}$ & $\begin{array}{l}\text { Comunidades que intentan } \\
\text { generar lecturas resistentes. }\end{array}$ \\
\hline & $\begin{array}{l}\text { Audiencia activa, genera } \\
\text { su propio significado. }\end{array}$ & Los fans reproducen el statu-quo. & $\begin{array}{l}\text { Las lecturas no siempre } \\
\text { son resistentes. }\end{array}$ \\
\hline & Visión idealizadora del fandom. & $\begin{array}{l}\text { Visión negativa y pesimista } \\
\text { del fandom. }\end{array}$ & $\begin{array}{l}\text { Una visión entre ideali- } \\
\text { zadora y negativa. }\end{array}$ \\
\hline \multirow[t]{3}{*}{ Objeto de estudio } & $\begin{array}{l}\text { Fan como individuo dentro } \\
\text { de una comunidad. }\end{array}$ & $\begin{array}{l}\text { Poco interés en el fan y } \\
\text { en las comunidades. }\end{array}$ & $\begin{array}{l}\text { Interés en el fan, la comunidad } \\
\text { y relación con otras estructuras. }\end{array}$ \\
\hline & $\begin{array}{l}\text { Fan como pieza clave } \\
\text { para resistencia. }\end{array}$ & $\begin{array}{l}\text { Fandom como un elemento } \\
\text { más del ecosistema mediático. }\end{array}$ & $\begin{array}{l}\text { Comprender la comunidad } \\
\text { dentro del ecosistema mediático. }\end{array}$ \\
\hline & $\begin{array}{l}\text { Interés en las motiva- } \\
\text { ciones individuales. }\end{array}$ & $\begin{array}{l}\text { Zero interés en las motiva- } \\
\text { ciones individuales. }\end{array}$ & $\begin{array}{l}\text { Interés en las motiva- } \\
\text { ciones individuales. }\end{array}$ \\
\hline
\end{tabular}

Tabla 1: PComparación entre las tres olas de fan studies. Fuente: Elaboración propia. 
La diferencia fundamental entre las dos es dónde se pone el foco de atención. Los académicos que han decidido acercarse a los fan studies mediante el análisis del contenido y del discurso parten de una premisa básica: "if fandom is to be taken seriously, then texts that fandom produce must be taken seriously as well" (Evans and Stasi 2014, 11). En este caso, el foco se sitúa en los productos generados por los fans, como por ejemplo los fan fictions, los manips, fan art, etc.

En los siguientes apartados profundizaremos en los intereses y objetivos de las investigaciones que utilizan métodos etnográficos, la evolución y diferentes debates alrededor de estos.

\section{La primera ola: las etnografías 'outsider'}

Tal y como hemos visto anteriormente, en los inicios de los fan studies los métodos etnográficos eran los más usados para analizar y entender a los fans. En la introducción de Textual Poachers, Henry Jenkins definía así el contenido del libro: "Textual Poachers offers an ethnographic account of a particular group of media fans, its social institutions and cultural practices" (1992, 1). Camille Bacon-Smith, en Enterprising Women, también se acerca a los fans desde una perspectiva etnográfica, estudiando las comunidades de fans de Star Trek. En el caso de Bacon-Smith (1992), su estudio se articula a partir de la perspectiva de género: el foco de interés de la autora son las comunidades de fans formadas principalmente por mujeres.

¿Por qué usar métodos etnográficos? Estos autores concebían el fandom como algo parecido a una red: "a collective identity and a mode of community organization" (Coppa 2014, 76), que de ninguna forma podía entenderse sin una metodología que contemplase el contexto y las relaciones de estas comunidades. Aunque el interés estaba en estas comunidades, sus prácticas, normas y códigos, también había interés en los textos que producían, ya que estos se entendían principalmente como formas de resistencia a los discursos mediáticos hegemónicos.

Autores como Sam Ford opinaban que este interés en el texto generaba jerarquías dentro de los fandoms, ya que algunos merecían más atención porque determinadas creaciones generaban más interés, ya sea por el nivel de destreza técnica que requieren o por el tipo de género del que eran fans, como podría ser la ciencia ficción $(2014,55)$.

Las actividades de los fans son heterogéneas, repartidas en varios niveles que van de más público a más privado, tanto en el ámbito digital como en el físico. Por ejemplo, tenemos las convenciones, que reúnen a muchos fans y, en general, son abiertas. Hay algunos eventos que están a medio camino, como pases de películas, y otras actividades más privadas y reducidas a pequeñas comunidades, como podría ser una partida de rol (Guitton and Cristofari 2017, 719).

Además, algunos de estos eventos se celebran de forma periódica y con cierta organización; otros son más espontáneos y de difícil acceso. Esto genera que las formas de estudiar determinados eventos puedan variar mucho y que las necesidades de cada investigación sean muy distintas. Debería, por lo tanto, ponerse el foco en esas prácticas quizás menos investigadas, ya sea porque son de más difícil acceso o no gozan del 'privilegio' al que hace referencia Paul Booth.

Más adelante hablaremos de las etnografías digitales o netnografías, pero en los primeros años de los fan studies, aunque ya empezaban a construirse espacios de fans en la red, internet no era una herramienta al alcance de toda la población y no estaba tan popularizado como sí lo estaría pocos años después. Por tanto, los primeros estudios se focalizaron especialmente en prácticas de fandom offline y luego fueron trasladando el interés a las prácticas online.

La etnografía es un método muy utilizado en los estudios culturales, aunque muchas veces se ha criticado que esta aproximación se emplea de forma poco rigurosa, o sin seguir los estándares de la antropología (Evans and Stasi 2014, 10). Michaela Di Leonardo define qué se entiende por etnografía desde la antropología y lo contrapone con las "etnografías" que se realizan en los estudios culturales. La autora considera que "'ethnography' has become a trendy methodological claim for some in cultural studies" $(2006,208)$.

De todas formas, los autores de la primera ola de los fan studies revindicaban los métodos etnográficos, aunque al mismo tiempo los cuestionaban. Jenkins, en la introducción de Textual Poachers apuesta por una observación participante, en la que se borren los límites entre etnógrafo y comunidad, generando así mucha más relación entre el investigador y los miembros del fandom y favoreciendo que los fans puedan cuestionar el relato que se genera sobre sus actividades (Jenkins 1992, 4).

En esta línea, el año 2002 surgió el concepto de "aca-fan", del que más adelante hablaremos en profundidad. Pero los primeros académicos de los fan studies practicaron lo que denominaron un 'outsider approach', que se hacía desde un punto de vista más impersonal y con más distancia (Meyer and Tucker 2007, 104).

Estas primeras aproximaciones etnográficas, caracterizadas por marcar la diferencia de roles y la intención del investigador de no presentarse también como 
fan, pueden surgir de una necesidad de legitimación de los estudios en los primeros años de la disciplina. De todas formas, se generaban tensiones. Camille Bacon-Smith, por ejemplo, cuando hizo la investigación que después publicaría en el libro Enterprising Women, sintió estas tensiones entre la comunidad de fans y su propia posición.

Este 'outsider approach' también ha resultado en la reticencia de muchos fans a participar en entrevistas o a dar su consentimiento para participar en estudios etnográficos, ya sea en un entorno digital o no. Katharina Freund comenta que sus encuentros con varios miembros de una comunidad de vidders explicitaba estas tensiones: "most were keen to participate but at the same time wary of my presence" (Freund and Fielding 2013, 331).

\section{Nuevas formas de relacio- narse: el 'aca-fan'}

Más adelante, en el año 2002, Matt Hills publicó el libro Fan Culture, donde acuñó el término "aca-fan", que proviene de 'academic' y de 'fan'. Aunque el término no aparece como tal hasta el año 2002, muchos académicos trabajando en los fan studies eran conscientes de su posición como fans, pero no había una palabra para definirlo ni tampoco un debate explícito sobre ello. Un "aca-fan" es, básicamente, todo aquel académico que también es fan, forme parte de la comunidad que estudia o no, o que simplemente es fan de otras cosas.

Henry Jenkins, en Textual Poachers, ya se presentaba como fan y como académico: "when I write about fan culture, then, I write both as an academic [...] and as a fan" $(1992,5)$. Lo que Hills define no es un concepto o una preocupación nueva, aunque en los primeros años de los fan studies esta cuestión no era central. Como hemos visto anteriormente, el académico quería separarse de su parte fan. A partir de cierto momento, los académicos ven la necesidad de reconocerse como fans, de incluir esa parte de ellos en la investigación.

¿Por qué este cambio? En primer lugar, la posición distante académica generaba problemas para adentrarse en comunidades de fans y generar confianza; presentarse como fan permitía tener el acceso y la complicidad de éstos. Además, significaba reconocer la realidad del académico, que se encontraba situado entre los dos mundos (Guitton and Cristofari 2017, 718).

A raíz de este cambio de concepción, también surge la autoetnografía. Esta práctica supone la autoreflexividad del aca-fan, que se relaciona de otra forma con la observación que está realizando, que en este caso se convierte en una observación participante. También permite que no se use la teoría para camuflar "personal attachments; challenges academic power and/or convention and treat the self and other identically" (Evans and Stasi 2014, 15).

Esta práctica empezó a popularizarse poco después de la publicación del libro de Hills (2002), ya que las autoetnografías permitían a los aca-fan reconciliarse con esta dualidad. Por ejemplo, Dianna Fielding cuenta lo siguiente sobre sus propias investigaciones en comunidades de fanfiction online: "I have been a fanfiction author for eight years [...] Many authors asked me to provide my online alias before they would

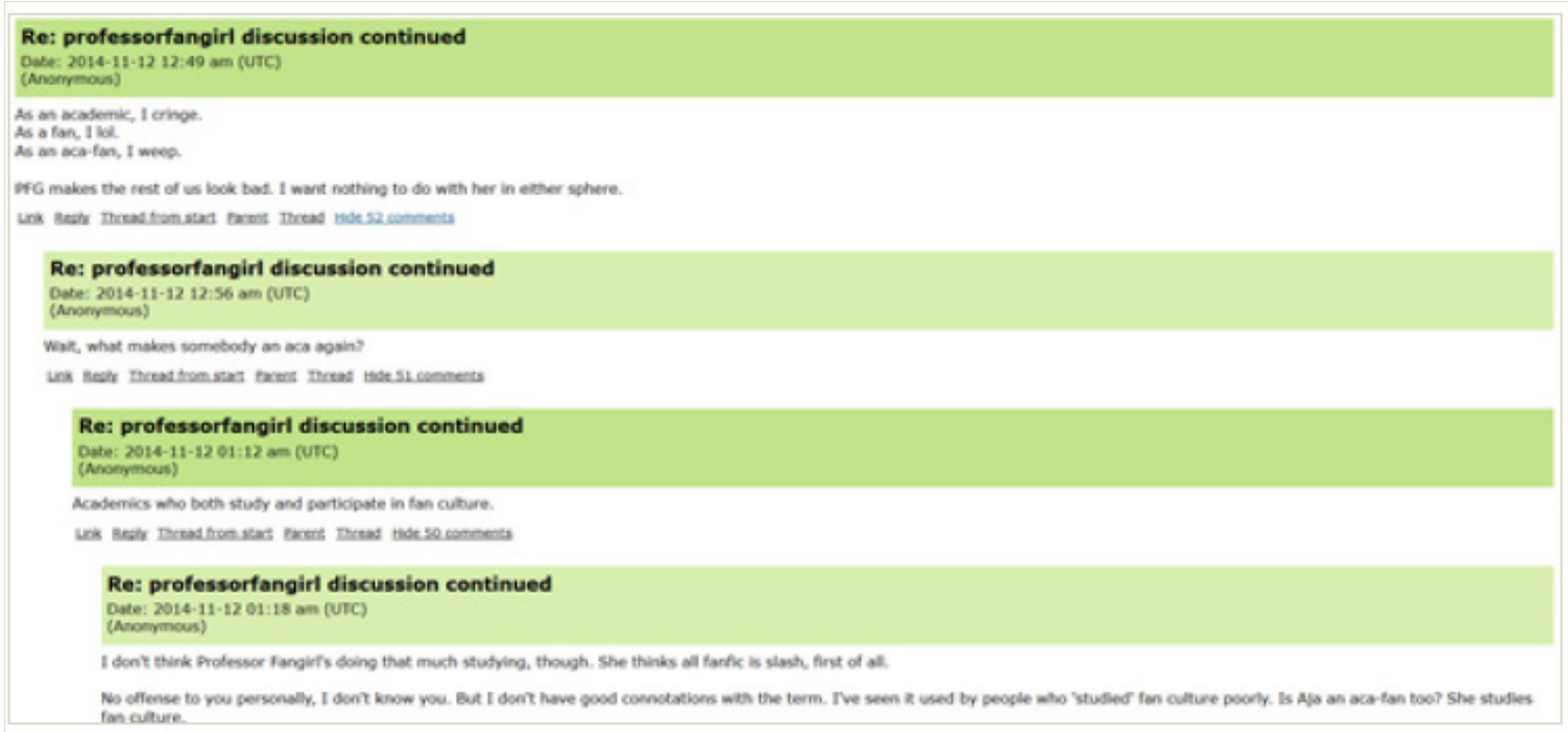

Figura 1. Captura de pantalla de un debate entre fans y aca-fan en un fórum (http://fail-fandomanon.dreamwidth.org/) 
agree to be interviewed. [...] I cannot be separated from my history as a fanfiction author" (Freund and Fielding 2013, 332).

No obstante, esta figura del aca-fan también presenta ciertas contradicciones y riesgos, especialmente en lo que se refiere a las jerarquías entre los fans y entre el académico. Aunque generalmente se cree que la figura del aca-fan ayuda a reducir esta diferencia, puede generar el efecto contrario: que el aca-fan aproveche su posición y privilegio de académico para conseguir acceso a ciertos ámbitos que estarían restringidos a un fan, como por ejemplo contacto con actores de un programa de televisión (Guitton and Cristofari 2017, 723). Además, surgen otros riesgos como el de reproducir conductas racistas, machistas, homófobas en los contextos de investigación ya que puede no producirse una reflexión crítica sobre la propia participación (Evans and Stasi 2014, 15).

Pero en general esta aproximación genera muchas más facilidades y, a la vez, que las investigaciones puedan ser más amplias y profundas. El aca-fan puede ya conocer de antemano el ámbito de estudio, y puede usar este conocimiento para adentrarse y estudiar de la mejor forma la comunidad. Y aunque no sea un estudio de su propio fandom, ya conoce ciertos códigos y prácticas similares en otros.

La posición del aca-fan puede ser complicada, ya que supone interactuar con dos mundos muy distintos. Mientras que el académico puede o debería considerarse como alguien más organizado, racional, analítico y con distancia respecto a eso que estudia, el fan tiene mucha más proximidad, es emocional y su relación con el objeto no está estructurada (Guitton and Cristofari 2017, 715). El aca-fan debe situarse entre

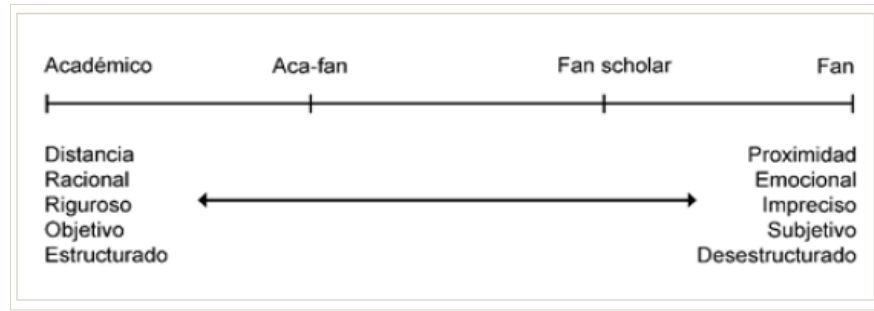

Figura 2. Diagrama sobre los distintos estadios entre el académico y el fan y los atributos de cada uno de ellos. Elaboración propia.

estos dos mundos, sin renunciar al rigor académico ni a la profesionalidad, pero sin alejarse de ciertas cualidades y características de los fans beneficiosas para la investigación.

Hay varios autores que consideran que la posición del aca-fan no debería limitarse a reconocerse a sí mismo como fan y académico a la vez, sino que debería trabajar para tener una relación más estrecha con el fandom y devolver parte de esta investigación e interacción a los propios fans (Booth 2013, 122). Esto en parte pasa por escuchar más la voz del fan y no solo la del investigador, pero también por hacer circular la información de las investigaciones entre estas comunidades.

\section{Fandom online: etnogra- fías digitales}

Esta relación más cercana y la eliminación de jerarquías entre fans y académicos se vuelve mucho más fácil y accesible gracias a internet y a los entornos digitales. Los fans rápidamente colonizaron internet, generando listas de correo, foros, páginas web, etc., donde encontrarse y compartir información. Obviamente, los académicos también pasaron a estudiar estas prácticas online, que en algunos aspectos no eran muy diferentes de las offline, pero en otros aspectos generaban dinámicas y espacios completamente nuevos.

Las comunidades online, por otra parte, ampliaban mucho más el número de personas dentro de un fandom, ya que mucha más gente tenía acceso a ellas, y no dependía de limitaciones geográficas, económicas... Además, para el investigador es mucho más fácil acceder a ellas. Por ejemplo, en lugar de tener que recopilar fanzines, puede entrar en una web como Fanfiction.net y consultar todos los fanfictions sobre determinado universo (Hellekson and Busse 2006, 76).

Además, la irrupción de internet y todas sus posibilidades también mejora la interconexión de los fans. Lucy Bennet identifica cuatro ámbitos en los que se benefician los fans: "(1) communication, (2) creativity, (3) knowledge and (4) organizational and civic power" $(2014,7)$. Los medios digitales permiten que estos cuatro ámbitos, ya presentes antes de internet, se amplifiquen, simplifiquen y multipliquen. Pongamos por caso la difusión del conocimiento: los fans también tienen acceso a muchas más producciones y otras visiones. Pueden compartirlo, generar wikis, newsletters, blogs...

De esta forma, las etnografías se trasladaron al mundo online, que permitía explorar ámbitos anteriormente de difícil acceso. También facilitaba un contacto más directo con los fans, aunque como hemos visto, les sigue generando cierta suspicacia.

Pero las etnografías digitales o netnografías también conllevan ciertos riesgos, debates y planteamientos especialmente a nivel ético. Son muchos los potenciales problemas que surgen, no solo en el ámbito de los fan studies sino de cualquier otra etnografía que explore una comunidad online. Aparecen temas de privacidad y recolección de datos, 


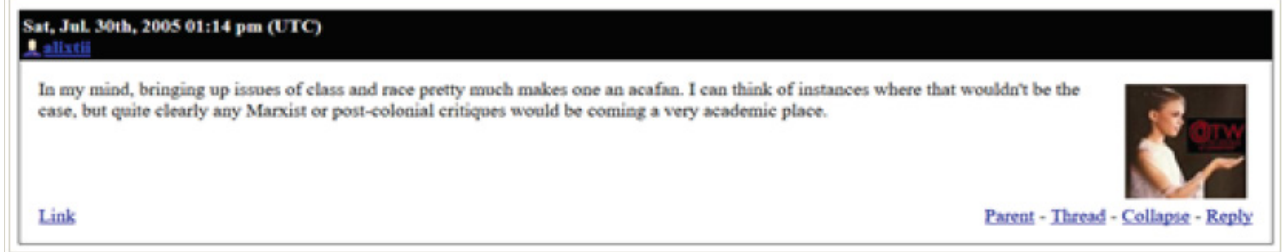

Figura 3. Comentario de un fan en un forum en el que se debatía la posición de los académicos y de los aca-fans (https://fanthropology.livejournal.com)

dificultades de establecer qué espacios son públicos o privados, la necesidad de consentimiento, etc.

En el caso de los fan studies esta cuestión de qué espacios son privados o públicos se difumina al trasladarlo al mundo digital. Mientras que en el mundo offline es más fácil hacer una distinción (una convención seria un evento público y quedar para hacer una partida de rol sería privado), es más difícil delimitarlo en las comunidades online. ¿Una web donde se cuelgan fan fictions es completamente pública? ¿Se tiene que pedir consentimiento a los autores (que publican bajo un pseudónimo) para usar sus historias? ¿Cómo se cita y se protege la privacidad de éstos? (Johnson, Lawson, and Ames 2018).

Todos estos elementos deben plantearse y debatirse entre los académicos de fan studies, así como de otras disciplinas que usen las etnografías digitales. Además, es crucial para mantener la confianza y complicidad de los propios fans, que podrían ver cómo sus publicaciones o creaciones se usan sin su consentimiento o conocimiento.

Todas las oportunidades y facilidades que el mundo digital nos aporta, sin ser apocalípticos, también vienen acompañadas de cuestiones éticas que no pueden ser ignoradas y que necesitan debatirse más allá de la pura aplicación metodológica. Por ejemplo, Matthieu Guitton y Cécile Cristofari se preguntan lo siguiente: "In a community where participation is largely defined by what people bring to it [...] is it ethical for a scholar to use the community for their academic work without contributing to it in their turn?" (2017, 726).

Estos y muchos más problemas necesitan un debate metodológico, que no solo se centre en los fan studies sino que observe más allá, en otras disciplinas que usen etnografías digitales y puedan encontrarse con los mismos problemas. Antes hemos mencionado la poca literatura que hay sobre metodología relacionada con los fan studies, pero en este caso es esencial debatir y proponer medidas y guías para recolectar información, cómo difundirla, etc.

Finalmente, una cuestión que surge a raíz de las etnografías digitales es que varios académicos creen que se está ignorando el mundo offline de los fandoms porque la mayoría de las investigaciones se han centrado en las comunidades y prácticas en internet. Lucy Bennet se cuestiona dónde quedan las prácticas offline y cómo estas se relacionan con las online, si es que tiene sentido separarlas. La autora cree que "despite the richness of the Internet and social media in allowing for creativity and communication to flourish further in fan cultures, 'offline activities are still evident" $(2014,15)$.

\section{Conclusiones}

Las formas de estudiar y aproximarse a los fans han ido mutando en el tiempo, ya sea por factores externos que supusieron un gran cambio - como la popularización de internet y el creciente número de comunidades de fans que la utilizan -, así como replanteamientos y debates dentro de los propios fan studies. Estas cuestiones más internas, de reflexión sobre la disciplina, por ejemplo, han resultado en nuevos conceptos como el de "aca-fan".

Todas estas evoluciones y cambios abren nuevos caminos, ámbitos y posibilidades de estudiar a los fans. El interés y la necesidad de estudiar a los fans sigue igual de vigente que cuando aparecieron los fan studies en los años 90. Estudiar a los fans significa estudiar "many of the key structuring mechanisms by which contemporary culture and society work; thus, the future of reception and audience studies requires thoughtful and innovative study of fans in all their forms, identities, media and spaces" (Sandvoss, Gray, and Harrington 2017, 16).

Pero tal y como apuntan Sandvoss, Gray and Harrington, es necesario discutir, hablar y plantear los métodos que usamos para estudiar a los fandom. De esta forma la disciplina puede afrontar adecuadamente retos éticos, que tal y como hemos visto, surgen en las etnografías digitales; o buscar puentes con otros estudios de fans, como podría ser con los sport fan studies para ampliar la visión y las formas de estudiar el fenómeno.

Otra de las cuestiones relevantes sería volver a poner el foco en las prácticas offline - sin olvidar las online - ya que muchas de las actividades de los fans siguen transcurriendo en entornos físicos y no solamente 
digitales (Duffett 2012; L. Bennett 2014), ya que centrar el foco solo en uno no consigue estudiar el fenómeno al completo.

De esta forma también se consigue superar uno de los problemas u obstáculos que más autores apuntan como una preocupación, que es la distancia entre los académicos y los fans. Por una parte esto provoca la dificultad de acceder a los fans, ya que se muestran reticentes y tienen cierta desconfianza hacia la figura del investigador (Freund and Fielding 2013). Pero lo más importante es reconocer la figura del aca-fan y no solo entenderla como la doble realidad del investigador sino como una forma de devolver la investigación a la comunidad estudiada.

Paul Booth propone tres ideas para potenciar el diálogo entre académico y fan, que serían: publicar más trabajos fan-friendly, participar en convenciones de fans y utilizar las redes sociales de una forma más pública y accesible $(2013,132)$.

Ideas como estas nos ayudan a replantearnos los fan studies y la figura del investigador, que, a pesar de tratarse de una disciplina con pocos años de vida, hechos como la aparición de internet y el cambio en el panorama mediático, nos obligan a replantearnos ciertas prácticas, que sin duda requieren debate y contraposición de ideas dentro de la comunidad académica, sin olvidar establecer un diálogo con los propios fans.

\section{Bibliografía}

Bacon-Smith, Camille. 1992. Enterprising Women: Television Fandom and the Creation of Popular Myth. Pennsylvania: University of Pennsylvania Press.

Bennett, Lucy. 2014. "Tracing Textual Poachers: Reflections on the Development of Fan Studies and Digital Fandom." The Journal of Fandom Studies 2 (1):5-20. https://doi.org/10.1386/jfs.2.1.5_1.

Bennett, Tony. 1998. "Cultural Studies: A Reluctant Discipline." Cultural Studies 12 (4):528-45. https://doi.org/10.1080/09502386.1 998.10383119.

Booth, Paul. 2013. "Augmenting Fan/Academic Dialogue: New Directions in Fan Research." Journal of Fandom Studies 1 (2):119-137. https://doi.org/10.1386/jfs.1.2.119_1.

Coppa, Francesca. 2014. "Fuck Yeah, Fandom Is Beautiful." The Journal of Fandom Studies 2 (1):73-82. https://doi.org/10.1386/ jfs.2.1.73_1.

Duffett, Mark. 2012. "Boosting Elvis : A Content Analysis of Editorial Stories from One Fan Club Magazine." Participations. Journal of Audience E Reception Studies 9 (2):317-36.

Evans, Adrienne, and Mafalda Stasi. 2014. "Desperately Seeking Methodology: New Directions in Fan Studies Research." Participations 11 (2):4-23. https://doi.org/DOI 10.1287/orsc.1070.0347.

Ford, Sam. 2014. "Fan Studies: Grappling with an 'Undisciplined' Discipline." The Journal of Fandom Studies 2 (1):53-71. https://doi. org/10.1386/jfs.2.1.53_1.
Freund, Katharina, and Dianna Fielding. 2013. "Research Ethics in Fan Studies." Participations. Journal of Audience E Reception Studies. http://www.participations.org/Volume\%2010/Issue\%201/16\%20 Freund\%20Fielding\%2010.1.pdf.

Guitton, Matthieu J, and Cécile Cristofari. 2017. "Aca-Fans and Fan Communities: An Operative Framework." Journal of Consumer Culture 17 (3):713-31. https://doi.org/10.1177/1469540515623608.

Harrington, C. Lee, and Denise D. Bielby. 2005. "Introduction: New Directions in Fan Studies." American Behavioral Scientist 48 (7):799800. https://doi.org/10.1177/0002764204273167.

Hellekson, Karen, and Kristina Busse. 2006. "Fan Fiction and Fan Communities in the Age of the Internet: New Essays." In Archontic Literature: A Definition, a History, and Several Theories of Fan Fiction, edited by Abigail Derecho, 61-78. Jefferson, NC: McFarland.

Jenkins, Henry. 1992. Textual Poachers: Television Fans E Participatory Culture. New York: Routledge.

---. 2006a. "Fan Studies." In Oxford Bibliographies in Cinema and Media Studies, edited by Krin Gabbard. New York: Oxford University Press.

---. 2006b. Fans, Bloggers, and Gamers: Exploring Participatory Culture. Fans, Bloggers, and Gamers: Exploring Participatory Culture. https://doi.org/10.1097/00002480-199909000-00019.

Johnson, Amy, Celeste Lawson, and Kate Ames. 2018. "Are You Really One of Us ? : Exploring Ethics, Risk and Insider Research in a Private Facebook Community," 102-9.

Leonardo, Michaela Di. 2006. "Mixed and Rigurous Cultural Studies Methodology - an Oxymoron?" In Questions of Method in Cultural Studies, edited by Mimi White and James Schwoch, 205-20. Blackwell Publishing.

Meyer, Michaela D. E., and Megan H. L. Tucker. 2007. “Textual Poaching and Beyond: Fan Communities and Fandoms in the Age of the Internet." Review of Communication 7 (1):103-16. https://doi. org/10.1080/15358590701211357.

Reijnders, Stijn, Koos Zwaan, Linda Dutis, and Abby Waysdorf. 2016. "Fandom." International Encyclopedia of Media Effects.

Sandvoss, Cornel, Jonathan Gray, and C. Lee Harrington. 2017. "Why Still Study Fans?" In Fandom: Identities and Communities in a Mediated World, 1-16.

Schimmel, Kimberly S., C. Lee Harrington, and Denise D. Bielby. 2007. "Keep Your Fans to Yourself: The Disjuncture between Sport Studies' and Pop Culture Studies' Perspectives on Fandom." Sport in Society 10 (4):580-600. https://doi. org/10.1080/17430430701388764. 

ISBN 978-84-09-20524-0



Máster Universitario en Investigación en Comunicación Social (MUCS)

\begin{tabular}{l|ll}
$\mathbf{u} p f$. & $\begin{array}{l}\text { Universitat } \\
\text { Pompeu Fabra } \\
\text { Barcelona }\end{array}$ & $\begin{array}{l}\text { DIGIDOC Grup de Recerca } \\
\text { en Documentació Digital } \\
\text { i Comunicació Interactiva }\end{array}$
\end{tabular} 